\title{
Eine bisher unbekannte Regentin des lateinischen Kaiserreiches.
}

Von

\section{Adolf Schanbe.}

Maria, D. g. imperitrix, bajula imperii Constantinopolitani, so nennt sich die Fürstin, die in einem an die Commune Pisa und das zeitweilige Oberhaupt derselben, den Podestà Ubald, gerichteten Schreiben ihren Dank für die trefflichen Dienste ausspricht, die der Vorsteher der pisanischen Colonie in Constantinopel, der Vicecomes Jacobus Scarlate, mit seinen Volksgenossen ihr und dem Reiche bisher erwiesen; unter Bestätigung der pisanischen Privilegien im romanischen Reiche ersucht sie die pisanische Regierung, ihrem Vertreter in Constantinopel, dem erwähnten Vicecomes, in Bezug auf seine erfolgreiche Thätigkeit einen Dank- und Aufmunterungsbrief zu senden.

Allgemein wird diese Maria für die ,schöne Bulgarin “, die Gemahlin Kaiser Heinrichs, jenes einzigen wirklich tüchtigen Herrschers, dessen das lateinische Kaiserreich sich zu erfreuen gehabt, gehalten und nur in Bezug auf den Atsatz des Jahres, dem diese Urkunde angehört, bestand bisher eine kleine Differenz. Das Schreiben ist nämlich nur nach Tag und Monat genau (vom 13. Februar) datiert; das Jahr ist nur durch die Indictionszahl $\alpha^{\prime}$ bezeichnet. Früher pflegte man die Urkunde in das Jahr 1214 zu setzen ${ }^{1}$, die beiden neuesten Herausgeber aber, Gius. Müller und der Graf Riant, nehmen, ohne sich über die Differenz zu äussern, in Rücksicht auf die Indiction das Jahr 1213 als das richtige an und dem ersteren folgt auch Heyd in seiner Geschichte des Levantehandels im Mittelalter 1, 321. Einige formelle Besonderbeiten der Urkunde baben nun den um die Geschichte der

1) So Tronci, Lünig, Buchon und nach diesem Hopf bei Ersch und Gruber, Encyclop. Art. Griechenland p. 246. 
Epoche der Kreuzzüge hochverdienten Herausgeber der Archires de l'Orient latin veranlasst, ein Facsimile derselben zu geben ${ }^{1}$ ); in der That kann angesichts desselben ein Zweifel in Bezug auf die Indictionszahl nicht aufkommen; der Buchstabe $\alpha$ ist mit aller wünschenswerten Deutlichkeit ausgeprägt und der Gedanke an einen Irrthum in der Kanzlei erscheint von vornherein ausgeschlossen.

Scheint so das Jabr 1213 entschieden den Vorzug zu verdienen, so erweckt diese Datierung doch in Bezug auf die Persönlichkeit der Kaiserin Maria ein erbebliches Bedenken. Die Vermählung Kaiser Heinrichs mit der Bulgarin, bisher in das Jahr 1214 gesetzt $^{2}$ ), müsste an den Anfang des Jahres 1213 oder gar in das Jahr 1212 zurückgerückt werden und es scheint nicht, dass dieser Ansatz den Angaben der Quellen gegeuüber aufrechterhalten werden könnte ${ }^{3}$ ). Dieser erste Zweifel erweckt andere. Welche Umstände sollen es denn gewesen sein, die die Bestellung der eben vermählten Kaiserin zur Reichsverweserin nothwendig gemacht haben könuten? Gerade für die Zeit Kaiser Heinrichs sind wir doch nicht so ganz unge nügend unterrichtet. Endlich, wie kommt Maria als stellvertretende Regentin dazu, die Privilegien Pisas zu bestätigen, da wir doch wissen, dass eine solche Bestätigung schon von Kaiser Heinrich selbst am Anfange seiner Regierung ausgesprochen worden war? ${ }^{4}$ )

Diesen Zweifeln und Bedenken gegenüber scheinen wir einen sicheren Anhalt für die Datierung der Urkunde an der Person des Adressaten, des Podestà Ubaldo, gewinnen zu müssen. Pflegten doch diese höchsten Beamten der italienischeu Städterepubliken zunächst nur für ein Jahr bestellt zu werden. Kein Zweifel nun, dass Tronci, der erste Herausgeber unserer Urkunde ${ }^{5}$, zur Wahl des Jahres 1214 lediglich durch den Umstand veranlasst worden ist, dass er von dem Adressaten zu wissen glaubte, dass er im Februar 1214 als Podestà von Pisa functioniert habe; die Augabe der Indiction hat er ganz ïbersehen. Nun stimmen in der That alle pisanischen Historiker darin überein, den Beginn der Amtszeit des Ubaldo Visconti (der übrigens nicht etwa, wie einige derselben angeben, dem bekannten mailändischen Geschlecht, sondern der mächtigen einheinischen Familie der

1) 'Tom. II, Documents, p. 256/7; ebenda die Angabe der früheren Drucke der Urkunde. $\left.\quad{ }^{2}\right)$ Hopf 1. c. $245 / 6$. 3) Vgl. namentlich den Zeitgenossen Robert de Clary bei Hopf, Chroniques Gréco-romanes $\$ 118 / 9$; Eracles lib. XXIX c. 12 im Recueil des historiens des croisades IL p. 289 ; auch Andr. Dandolo setzt die Heirat in das 9. Jahr des Dogen Pietro Ziani, also in das Jahr 1214. 4) Gius. Müller, Documenti sulle relazioni delle città Toscane coll' Oriente p. 86/7; vgl. p. 437. 5) Memorie istoriche di Pisa, Livorno 1682, p. 177. 
Visconti angehörte) in das Jahr 1214 zu setzen, und ihre Angaben gehen auf alte Beamtenverzeichnisse zurück, von denen uns eins in dem von Bonaini veröffentlichten Memoriale Consulum et Potestatum vorliegt, das seine Aufzeichnungen mit Ubaldo Visconti beginnt und diesem eine Amtsdauer von 2 Jahren, rom Jahre 1214 angefangen, beilegt 1). Nun würden die Verfechter des Jahres 1213 allerdings geltend machen können, dass diese einheimisch pisanischen Quellen sich vermuthlich auch des calculus pisanus bedient haben werden, der unserer Zeitrechnung bekanntlich um ungefähr $3 / 4$ Jahre voraus ist; die Angabe des Memoriale würde also gerade auf das wirkliche Jahr 1213 zu beziehen sein. Der Calcul ist richtig; aber das Menoriale selbst wie alle anderen pisanischen Quellen beflnden sich im Irrthum; der consensus omnium ist diesmal nur ein error omnium. In Wahrheit ist nämlich Ubaldo Visconti weder im Jahre 1213, noch im Jahre 1214 Podestà von Pisa gewesen, wie sich aus urkundlichen Belegen mit voller Sicherheit erweisen lässt.

Zunächst für das Jahr 1214. Der zeitgenössische Anualist Genuas, Ogerius Panis - pisanische Annalen fehlen in diesem Zeitraum redet unter diesem Jahre von Consules Pisanorum in solcher Art, dass das gleichzeitige Vorhandensein eines pisanischen Podestà durchaus nusgeschlossen erscheint ${ }^{2}$ ). Ueberdies ist uns vom Juni/Juli desselben Jahres der Vertrag Pisas mit Venedig erhalten, der auf pisanischer Seite vou den vier namentlich genannten Consuln der Republik, die in derselben Orkunde auch als rectores Pisane civitatis bezeichnet werden, abgeschlossen wird ${ }^{3}$ ). Wollten wir nun dem calculus pisanus zu Liebe den Beginn der Amtszeit Ubaldo's in das Jahr 1213 unserer 7eitrechnung setzen, so würden wir mit den pisanischen Quellen doch in Widerspruch gerathen, da dieselben dem Podestat Ubaldo's sämmtlich eine Dauer von zwei oder drei Jahren beimessen. Dieser indirecten Beweisführung kann ich aber auch den positiven Nachweis zur Seite stellen, dass Ubaldo auch im Jahre 1213 nicht Podestà gewesen ist; in Bonainis Sammlung pisanischer Documente, die ich im Archivio di Stato zu Pisa einzusehen vor längerer Zeit Gelegenheit hatte, beflndet sich eine noch ungedruckte Urkunde vom 24. März 1213, aus der hervorgeht, dass auch zu diesem Zeitpunkte ein Collegium von vier Rectores an der Spitze des pisanischen Staatswesens gestauden hat.

Nach diesen urkundlichen Zeugnissen muss also in den pisanischen Beamtenverzeichnissen ein Fehler vorliegen und wir sind auch

ग) Archivio stor. ital. serie I, vol. VI, parte 2, p. $648 . \quad$ 9) Mon. Germ. SS. 18, 134 . 3) Gius. Müller, l. c. 88. 
im Stande, die Entstehung desselben in dem Memoriale Consulum et Potestatum zu erkennen. In dieser Quelle folgt nämlich auf das Podestat Ubaldo's, das mit zweijähriger Dauer von 1214 ab angesetzt wird, unmittelbar das Consulat des Bulsus und Genossen im Jahre 1218. Die scheinbar hier vorbandene Lücke verschwindet, wenn wir statt der Jabreszahl MCCXIV des jetzigen Textes mit leichter Correctur MOCXVI lesen. Der Fehler ist offenbar sehr früh entstanden und so in alle spätereu Quellen und Darstellıngen der pisanischen Geschichte übergegangen. Das MCCXVI des pisanischen Memoriale entspricht nun dem Jahre 1215 der gewöhniichen Zeitrechnung und in Wirklichkeit ist Obaldo Visconti in den Jahren 12151217 Podestà vou Pisa gewesen, was ich hier als nicht weiter zur Sache gehörig nur beiläufig bemerken will.

Wenn wir uns nun, nachdem die bisherigen Ansätze des Schreibens der Reichsverweseriu Maria sich als hinfällig erwiesen haben, dem genaueren Wortlaut der Urkunde selbst zuwenden, so werdeu wir billig in ein gelindes Erstauneu darüber gerathen, dass mau überhaupt jemals auf den Gedanken kommen kounte, in dieser Maria die Gemahlin Kaiser Heiurichs zu erblicken. In dem Schreiben spricht sie der Commune Pisa ibren Dank ans ,de servitio et honore, que nobis vir nobilis D. Jacobus Scarlate, vicecomes vester Pisanorum in Constantinopoli, necnon et fratrinostror[egi] recordationis inclite, quondam imperatori Constantinopolitano, ac eius predecessoribus, tamquam vir providus et discretus, cum toto communi suo impendere non cessavit " und weiterhin bestätigt sie den Pisanern die Privilegien "que tam abipso fratre uostro quama predecessoribus...obtinuerant." Es genügt diese Worte hierher zu setzen, um die pure Unmöglichkeit der bisherigen Auffassung darzuthun'). Die Maria unserer Urkunde fungirt als Reichsverweserin nach dem Tode ihres Bruders, der Kaiser von Constantinopel gewesen; das geht doch klar aus der Urkunde hervor. Räthselhaft bleibt dabei zunächst, wie sie als eine Schwester des verstorbenen Kaisers sich doch selbst als Kaiserin bezeichuen kann.

1) Tronci, der erste Herausgeber der Urkunde, liest: necnon et fratri nostro. et recordationis inclitae quondam imperator Constantinus, et eius praedecessoribus; das war dann freilich völlig unverständlich. Lünig, der das Original oftenbar gar nicht gesehen und die Urkunde allein auf die Autorität Tronci's hin, allerdings obne diesen zu nenuen, in seinem Cod. dipl. Italiae 1, 1061/2 aufgenommen hat, glaubte die Stelle dadurch zu bessern, dass er aus praedecessoribus ein praedecessores machte. An der zweiten oben citierten Stelle hat Tronci und demgemäss auch Lünig statt fratre nostro das völlig verkehrte vestro. 
Ist nun an die Gemahlin Kaiser Heinrichs in keinem Fall zu denken, hat sich von den wenigen Jahren erster Indiction, die in den kurzen Zeitraum der Existenz des lateinischen Kaiserreiches fallen, das erste als für unsere Zwecke uubrauchbar erwiesen, so bleibt uns nur übrig, zu versuchen, ob nicht das nächste Jahr derselben Indiction, das Jahr 1228, den Voraussetzungen unserer Urkunde besser entspricht.

Da ist nuu zunächst ron entscheidender Wichtigkeit, dass in diesen Jahre Ubaldo Visconti wirklich Podestà von Pisa gewesen ist; ausser in den Jahren 1215 - 1217 war er auch 1227 und 1228 mit der obersten Leitung der Republik betraut, wie die pisauischen Quellen in voller Uebereinstimmung, und diesmal mit Recht, angeben 1). Das Jahr 1228 ist das einzige Jahr der ersten Indiction, in dem Ubaldo Podesta gewesen; in dieses Jahr muss also unsere Urkunde gebören, wenn die Indiction nicht falsch oder gar die ganze Urkunde gefälscht ist. $\mathrm{Zu}$ solchen Annahmen liegt indessen so lange kein Grund vor, so lange die von unserer Urkunde vorausgesetzten Verhältnisse sich dem Jahre 1228 einfügen lassen, ohne mit anderweitig beglaubigten Thatsachen in Widerspruch zu gerathen.

Nun erfüllt das Jahr 1228 auch die zweite unerlässliche Vorbedingung: der Thron von Constantinopel war durch den Tod Kaiser Roberts erledigt. Hilfeflehend hatte er sich nach Rom zu Papst Honorius III. begeben; auf der Rückreise ereilte den schwachen Fürsten bei seinem Schwager Villehardouin in Achaja der Tod ${ }^{2}$ ). Unsere vom 13. Februar datierte Urkunde berechtigt uns nun schov, zu schliessen, dass der Tod des Kaisers im Anfang des Jabres 1228, vielleicht sogar woch im Jahre 1227 eingetreten ist; war er doch, soviel wir wissen, noch bei Lebzeiteu Honorius' III. ( $\dagger$ 18. März 1227) von Rom abgereist.

Es scheint uns nur noch übrig 211 bleiben, auch die Schwester Kaiser Roberts ausfindig zu machen. Nun hatte Kaiser Robert in der That eine Schwester Namens Maria; ihre umsichtige Mutter Jolanta hatte sie im Jahre 1218 an Theodor Laskaris, den Kaiser von Nicaea, verheirátet und so ein freundschaftliches Verhältnis der beiden rivalisierenden Reiche anzubahnen gesucht; doch schon im Jabre 1222 raffte den tüchtigen Herrscher der Tod dahin. Diese Maria, Laskaris Witwe, mussalso die Regentin unserer Urkundesein.

Diesem Resultat steht nun keine geringere Schwierigkeit entgegen, als die, dass Maria in dieser Zeit selbst nicht mehr zu den Lebenden

1) So das Memoriale, 1. c.; der Anonymus bei Muratorí, Rer. It. SS. 24, 64: ; die Chroniche di Pisa bei Tartinius 1, 497 ; Tronci p. 184; Roncioni, istorie pisane ed. Bonaini im Arch. stor. ital. VI, 1, 49.. 2) Hopf S. 259. 
gehört haben soll. Hopf erzählt uns nämlich, Du Cange folgrend, S. 249: ,Da starb Theodor und bald nach ihm seine Gemahlin Marïa von Courtenay "; wenn das richtig oder ganz wörtlich zu nehmen wïrie, so würden wir allerdings trotz allem unser Ergebnis fallen lassen müssen. Die Notiz beruht auf Nikephorus Gregoras, byz. hist. lib. II cap. 1, wo er nach der Erzählung von Laskaris' Tode fortfïhrt: $x \alpha \tau x$ -

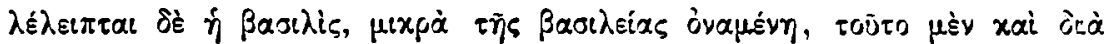

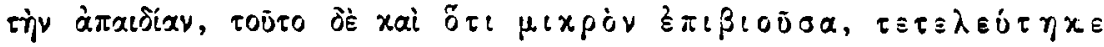
$x \propto i$ aù $\tau \dot{\eta}$. Nun ist die Autorität dieses Schriftstellers für unsere Zeit uicht gerade hoch anzuschlagen. Ist doch sein die Jahre 1204-1351 umfassendes Werk erst mehr als ein Jahrhundert später eutstanden. Auch liebt es der Autor sehr, sich die Dinge in seiner Art zurechtzulegen und den Bericht über Thatsachen durch Hinzufügung ron Gründen, die ihm plausibel erscheinen, zu erweitern. So erzählt er z. B. von derselben Maria am Ende des ersten Buches, dass sie von Laskaris keine Kinder hatte und fügt nun zur Motivierung hinzu, , sei es, weil sie von Natur unfruchtbar war, sei es, weil der frühe Tod des Kaisers ihr die Möglichkeit des Kindergebärens raubte "! Aehnlicher Art scheint mir auch seine obige Bemerkung. Ich meine nicht, dass er eine Notiz über den Torl der Kaiserin vor sich hatte. In seiner umständlichen Art aber empfand er das Bedürfnis, sich mit der Thatsache, dass Laskaris eine Witwe hinterliess, irgendwie abzufinden und da er Maria weiterbin nicht mebr erwähnt fand, schien es ihm am einfachsten, den baldigen Tod derselben anzunehmen. Wie wenig wir auch sonst berechtigt sind, einzelne Ausdrücke unseres

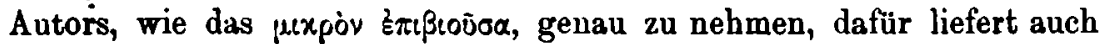
folgende für uns noch aus anderem Grunde beachtenswerthe Stelle einen Beleg. Bekanntlich hatte sich nach Laskaris' Tode unter Verdrängung seines unmündigen Sohnes (aus der Ehe mit der armenischen Prinzessin Philippa) sein Schwiegersohn, der thatkräftige Johanues Vatatzes, ein entschiedener Gegner der Lateiner, der Herrschaft bemächtigt. Nikephorus erzählt uns nun, dass auch zwei Brüder des verstorbenen Kaisers, Alexius und Isaak, vor Vatatzes flüchteud sich nach Constantinopel begeben hätten, wo damals Kaiser Robert regiert

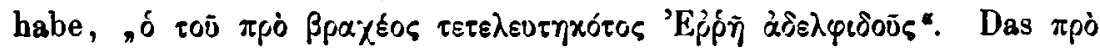

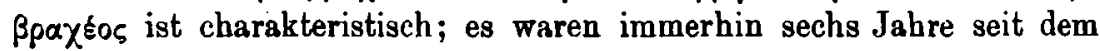
Tode Kaiser Heinrichs verstrichen und eines längeren Zeitraunes bedürfen wir auch nicht, um Maria bei dem Tode ihres Bruders Robert noch am Leben sein zu lassen. Auf keinen Fall also ist es gerecht-

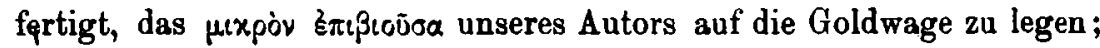
wenn unserer Annahme kein anderes Bedenken im Wege steht, so 
werden wir im Rechte sein, die Angabe dieses späten Schriftstellers als eine mehr zufällig gethane Aeusserung anzusehen, der er selbst keinerlei grössere Bedeutung beimass, und dieselbe üher Bord zu werfen.

Ueberlebte nun Maria ihren verstorbenen Gemahl längere Zeit, so ist es sehr begreiflich, dass an dem neuen Hofe von Nicaea kein Platz für sie war; wenn es nicht schon eher geschehen, so wird sie doch sicher mit den beiden oben genannten Brüdern ihres Gemahls den Hof von Constantinopel, wo ihr Bruder regierte, aufgesucht haben. Es erscheint ferner durchaus in den Verhältnissen begründet, wenn wir nun weiter annehmen, dass Kaiser Robert bei seiner Abreise nach Italien seine nächste Verwandte, seine Schwester Maria, für die Dauer seiner Abwesenheit zur Reichsverweserin bestellt habe. Es ist endlich natürlich, dass sie auch nach dem Tode ihres Bruders, der nur einen unmündigen Sohn, den nachmaligen Balduin II., hinterlassen, die Regentschaft zunächst noch fortgeführt haben wird. In dieser Stellung sandte sie am 13. Februar 1228 unser Schreiben nach Pisa. Am Ende desselben Jahres begegnen wir allerdings einem andern Reichsverweser; am 11. December 1228 schliesst Narjaud de Toucy als bajulus imperii Romanie im Namen der Barone mit Theodor von Thessalonich Waffenruhe auf ein $\mathrm{J}_{\mathrm{ahr}}{ }^{1}$ ). Ein solcher Wechsel in der Regentschaft kann indes vicht Wunder nehmen. Die Stellung Marias bernhte auf dem ihr von ihrem Bruder ertheilten Mandat, das sich nur auf die Zeit seiner $A b$ wesenheit bezog; für den Fall, dass nach dem Tode des Herrschers die Einsetzung einer Regentschaft nothwendig war, übten die Barone das Wahlrecht aus; die Principes et Barones et Milites, wie sie in einer analogen Urkunde genannt werden ${ }^{2}$ ). Den Baronen mochte die Fortdauer der Regentschaft einer Frau in der bedrängten Lage, in der das Reich sich befand, nicht genehm sein. Vielleicht bängt der Wechsel in der Regentschaft aber auch mit den grossen Gegensätzen der Zeit zusammen. Eine Partei am Hofe der Lateiner wünschte die Regentschaft dem bulgarischen Czaren, dem trefflichen Johann Asen II. zu übertragen, zumal dieser durch seine Vermähluug mit einer Tochter des Königs von Ungarn, einer Nichte Roberts, mit dem Kaiserhause in naher Verbindung stand. Die clericale Partei widerstrebte mit Erfolg und der Einfluss Gregors IX. war es bekanutlich, der schliesslich die Wahl jenes Johann von Brienne durchsetzte, der den gebannten Staufenkaiser im Sommer 1228 in Apulien bekämpft hat. Dass Maria der ersten Partei angehört haben wird, ist schon der verwandtschaftlichen Verhältnisse wegen anzunehmen; und in diesem

1) Hopf S. 252. 2) Tafel und Thomas, Urk. zur alteren Handels- und Staatsgeschichte der Republik Venedig 2, 88. 
Zusammenhange würde ein neues Licht darauf fallen, dass sie in ihrem Schreiben gerade an die Pisaner, die treuesten unter den Anhängern des gebannten Kaisers, Anlehnung sucht und sie bittet, ihrem Vertreter in Constantinopel Anerkennung für seine bisherige Thätigkeit in ihrem und des Reichs Interesse zu zollen, ut suus erga nos et imperium augeatur animus, et augendo bonum propositum duplicetur, cum nullus utilior aut necessariornobis et imperio esse possit."

Dem sei indes wie ihm wolle; es bleibt uns noch übrig, dariuf hinzuweisen, dass unsere Ansetzung und Auffassung der Urkunde auch alle anderen bisher hinsichtlich derselben aufgetauchten Bedenken zerstreut. Die Bestätigung der Privilegien der Pisaner durch die Regentiu hat in dieser Zeit, wo es dem Reiche thatsächlich an einem andern Oberhaupte fehlte, einen wirklichen Sinn; auf der anderen Seite entspricht es dem provisorischen Charakter dieser Regentschaft durchaus, dass diese Bestätigung in wenig solenner Form und mehr beiläufig ausgesprochen wird. In ungeahnter Weise hat sich nun auch das Räthsel in der Titulatur der Regentin gelöst. Wenn sie sich Maria, D. g. imperatrix nennt; so thut sie das keineswegs als Kaiserin von Constantinopel, wie man im ersten Augenblick natïrlich vorauszusetzen geneigt ist, sondern als Kaiserin-Witwe von Nicaea, während sie für das lateinische Kaiserreich eben nur als bajula fungirt. Und dass sie nur diese Stellung in Constantinopel einnimmt, das erklärt wieder die diplomatische Eigenthümlichkeit der Urkunde, die dem Grafen Riant aufgefallen ist; da sie nicht Kaiserin von Constantinopel war, so hatte sie in der That kein Anrecht auf das xavixdzcov.

Auch für die bessere Lesung der Urkunde selbst fällt schliesslich noch ein kleiner Gewinn ab. In den Worten "fratri nostro r[egi], recordationis inclite, quondam imperatori Constantinopolitano ", wird man bei genauerer Erwägung das regi schwerlich für eine glückliche Ergänzung halten. Zwar das Anfangs-, $r$ ", das Tronci einst für "et "las, ist deutlich erkennbar; auch der Raum würde durch regi ausgefüllt werden, aber man erwartet an dieser Stelle doch nicht den Titel des Bruders, der ja überdies hinterher angegeben wird, sondern seinen $\mathrm{Na}$ men. Ich meine also, dass wir ein in Abbreviatur geschriebenes Roberto zu ergänzen haben; die Spuren des ${ }^{b}$ " glaube ich in dem Facsimile noch zu erkennen. Wäre dies eine Wort vicht zufällig unleserlich geworden, so hätte der Irrthum bezüglich der Persönlichkeit der Regentin Mari. schwerlich entstehen, noch weniger so lange sich behaupten können. 